\title{
The interaction between gaze and facial expression in the amygdala and extended amygdala is modulated by anxiety
}

\author{
Michael P. Ewbank ${ }^{1}$, Elaine Fox ${ }^{2}$ and Andrew J. Calder ${ }^{*}$ \\ Medical Research Council Cognition and Brain Sciences Unit, Cambridge, UK \\ Department of Psychology, University of Essex, Essex, UK
}

\section{Edited by:}

Patrik Vuilleumier, University Medical Center and University Hospital Geneva, Switzerland

\section{Reviewed by:}

Nathalie George, Centre National de la Recherche Scientifique, France;

University Pierre and Marie Curie, France

Reginald Adams, Pennsy/vania State University, USA

\section{${ }^{*}$ Correspondence:}

Andrew J. Calder and Michael P. Ewbank, Medical Research Council Cognition and Brain Sciences Unit, 15 Chaucer Road, Cambridge CB2 7EF, UK.

e-mail: andy.calder@mrc-cbu.cam.ac. uk and michael.ewbank@mrc-cbu.cam. ac.uk
Behavioral evidence indicates that angry faces are seen as more threatening, and elicit greater anxiety, when directed at the observer, whereas the influence of gaze on the processing of fearful faces is less consistent. Recent research has also found inconsistent effects of expression and gaze direction on the amygdala response to facial signals of threat. However, such studies have failed to consider the important influence of anxiety on the response to signals of threat; an influence that is well established in behavioral research and recent neuroimaging studies. Here, we investigated the way in which individual differences in anxiety would influence the interactive effect of gaze and expression on the response to angry and fearful faces in the human extended amygdala. Participants viewed images of fearful, angry and neutral faces, either displaying an averted or direct gaze. We found that state anxiety predicted an increased response in the dorsal amygdala/substantia innominata (SI) to angry faces when gazing at, relative to away from the observer. By contrast, high state anxious individuals showed an increased amygdala response to fearful faces that was less dependent on gaze. In addition, the relationship between state anxiety and gaze on emotional intensity ratings mirrored the relationship between anxiety and the amygdala/SI response. These results have implications for understanding the functional role of the amygdala and extended amygdala in processing signals of threat, and are consistent with the proposed role of this region in coding the relevance or significance of a stimulus to the observer.

Keywords: emotion, anxiety, fMRI, face processing, amygdala, expression, gaze

\section{INTRODUCTION}

Facial expressions of both anger and fear represent potent signals of threat. Across various species, angry expressions are commonly used in face-to-face encounters in order to control or change the behavior of others via the assertion of authority; as when warding off competitors for valued assets, such as food, resources, social status or territory (Averill, 1982; Blanchard and Blanchard, 2003). By contrast, fear is seen as a more reflexive response to threat that has the function of signaling danger and thus serves to increase the vigilance of both the expresser and the observer (Phelps et al., 2006; Susskind et al., 2008).

The primary role of angry expressions in controlling or manipulating others' behavior (Averill, 1982; Blanchard and Blanchard, 2003) suggests that the level or relevance of threat signaled by this expression may be particularly dependent on where the expresser is looking. Indeed, numerous behavioral studies suggest that perception of anger in faces is enhanced when their gaze is directed at the observer (Adams and Kleck, 2005; Graham and LaBar, 2007; Sander et al., 2007; Bindemann et al., 2008). The influence of gaze on the perception of fearful faces is less clear. Some behavioral studies indicate that the processing of fearful faces is enhanced by averted relative to direct gaze (Adams and Kleck, 2005; Sander et al., 2007), while others have found mixed effects (Graham and LaBar, 2007; Hess et al., 2007; Bindemann et al., 2008).

The amygdala and extended amygdala have a prominent role in the processing of threat-related stimuli such as fear and anger (Calder et al., 1996, 2001; Adolphs, 1999). The extended amygdala is charac- terized as continuous structures from the central and medial nuclei of the amygdala to corresponding regions of the bed nucleus of the stria terminalis, projecting through a region known as substantia innominata (SI) (Heimer et al., 1997; Heimer, 2003). The SI also appears sensitive to facial expressions of threat, and has been proposed to play a specialized role in processing the arousal or salience of facial expressions (Kim et al., 2003; Davis et al., 2009). A number of studies have investigated the way in which the response to facial signals of threat in the amygdala and extended amygdala is influenced by gaze direction, to date however, findings have been mixed. An initial fMRI study by Adams et al. (2003) found a greater amygdala response to angry faces with averted gaze and fearful faces with a direct gaze, relative to direct gaze anger and averted gaze fearful faces, respectively (Adams et al., 2003). This was attributed to the idea that direct gaze fearful faces and averted gaze angry faces constitute more ambiguous signals of threat than their averted and direct gaze counterparts; supporting the role of the amygdala in coding signals of ambiguous threat (Davis and Whalen, 2001; Whalen, 2007). However, subsequent studies have found mixed results. A study by Sato et al. (2004) found an increased response to angry faces directed at, rather than away from the observer (Sato et al., 2004). By contrast, Straube et al. (2010) found that angry faces produced an enhanced amygdala response for averted relative to direct gaze expressions, consistent with the findings of Adams et al. (2003). In addition, Hadjikhani et al. (2008) found an increased response to averted gaze fear faces, contrasting with the findings of Adams et al. (2003). As discussed 
earlier, one reason for these inconsistent results may relate to the variable influence of gaze on the processing of fearful faces. However, a second issue is that these studies have not accounted for the marked influence of individual differences in anxiety on the response to facial signals of threat, as found in numerous behavioral studies (Bar-Haim et al., 2007) and more recent neuroimaging research (Bishop et al., 2004; Etkin et al., 2004; Ewbank et al., 2009c).

These latter studies provide consistent evidence that individual differences in anxiety in a healthy, non-clinical population show a positive correlation with the amygdala response to facial signals of fear (Bishop et al., 2004; Etkin et al., 2004; Ewbank et al., 2009c) and angry faces (Ewbank et al., 2009c). However, this relationship is differentially modulated by the observer's focus of attention. Heightened levels of anxiety are associated with an increased amygdala response to fearful faces whether attended or not, although the relationship was more marked for unattended faces (Bishop et al., 2004; Ewbank et al., 2009c), whereas a corresponding amygdala correlation for angry faces is only found when the faces are attended (Ewbank et al., 2009c). This differential effect of attention accords with the qualitatively different forms of threat signaled by these two expressions (Ekman and Friesen, 1975) - angry expressions are commonly used to exert authority in face-to-face encounters, whereas fearful expressions are primarily used to signal the presence of danger within the environment.

Whereas the aforementioned study shows that the observer's level of anxiety and focus of attention have a clear influence on the neural response to facial signals of threat, an equally important question concerns how the expresser's direction of attention affects the neural response to these facial signals in high and low-anxious participants. As gaze direction is an important social cue, this is likely to be strongly modulated by individual differences in anxiety. Of particular relevance to the current study, Hess et al. (2007) showed that angry faces directed towards, relative to away from the observer produce increased self-reported anxiety in observers. In contrast, direct and averted fearful faces were rated as being equally anxiety-provoking (Hess et al., 2007). High-anxious individuals also show a more delayed disengagement of attention from angry faces with direct gaze relative to neutral, happy, or fearful faces with a direct gaze, and enhanced gaze cueing effects from fearful faces (Fox et al., 2007). These findings indicate that anxiety and gaze direction may have a central role in determining the behavioral and neural response to facial signals of threat (see also Putman et al., 2006; Tipples, 2006) for enhanced cueing to fearful faces.

In the current study we used fMRI to examine the influence of direct and averted gaze direction on the amygdala response to angry, fearful, and neutral faces, paying particular attention to the effects of individual differences in anxiety. In other words, the expectation was that anxiety would correlate with an increased response in the amygdala and extended amygdala to angry faces gazing towards but not away from the observer, while the response to fear would be less dependent upon gaze direction.

\section{MATERIALS AND METHODS PARTICIPANTS}

Thirty-one healthy volunteers ( 14 female, all right-handed, aged 18 - to 35-years old, mean age $=25.2$ ) with normal, or corrected to normal vision participated in this study. None had a history of neurological disease or head injury or were currently on medication affecting the central nervous system. The data from four participants were excluded due to excessive movement or signal dropout. The study was approved by Cambridgeshire Local Research Ethics Committee. All volunteers provided written informed consent and were paid for participating.

Participants completed the Spielberger State-Trait Anxiety Inventory (STAI) (Spielberger, 1983). Participants' state anxiety scores ranged from 20 to 39 (mean $=29, \mathrm{SD}=5.3)$, and trait anxiety scores from 21 to 62 (mean $=37, \mathrm{SD}=9.3)$. These scores are similar to published norms for this age group (Spielberger, 1983). As expected, state and trait anxiety scores were correlated $(r=0.50$, $P<0.01)$. Given that recent evidence has shown social anxiety influences the neural response to fearful and angry faces (Phan et al., 2006; Evans et al., 2007) we also sought to account for the influence of this dimension. Participants therefore completed the Brief Fear of Negative Evaluation (FNE) revised scale, a measure of sensitivity to negative evaluation by others and avoidance of social-evaluative situations (Carleton et al., 2006). Participant's FNE scores ranged from 1 to $44($ mean $=24.1, \mathrm{SD}=11.3)$, also similar to published norms (Carleton et al., 2007). All measures were completed before the scanning session.

\section{TASK DESIGN}

Participants lay supine in the magnet bore and viewed images projected onto a screen visible via an angled mirror placed above the participant's head. The face stimuli comprised grayscale pictures of neutral, fearful and angry facial expressions, posed by 10 different individuals ( 5 males, 5 females) taken from the NimStim Face Stimulus Set (Tottenham et al., 2009) and the Karolinska Directed Emotional Faces (KDEF) image set (Lundqvist and Litton, 1998). Faces were cropped to an elliptical shape to eliminate hair and background cues (See Figure 1). Gaze direction was manipulated using Adobe Photoshop (http://www.adobe.com/), as in previous studies

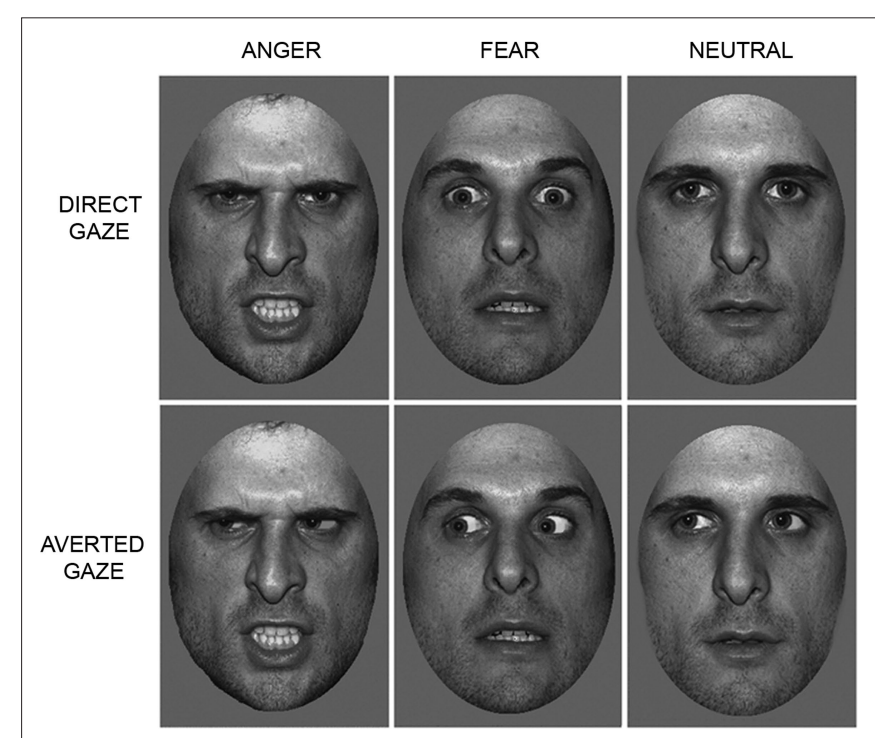

FIGURE 1 | Trials consisted of images of angry, fearful or neutral faces, displaying either a direct or averted gaze. 
(Adams et al., 2003; Adams and Kleck, 2005; Hadjikhani et al., 2008; Ewbank et al., 2009b). Each face subtended a visual angle of approximately $8^{\circ} \times 5^{\circ}$. Presentation of images was controlled using E-Prime software (Psychology Software Tools Inc., Pittsburgh, PA, USA).

Trials were presented in a pseudo-randomized order generated using the "Mix" randomization tool (Van Casteren and Davis, 2006). Participants were required to categorize the gender of each face and were instructed to respond as quickly and accurately as possible via a button press. Reaction time and accuracy data were recorded. Each face was presented for a period of $1000 \mathrm{~ms}$, followed by a variable inter stimulus interval of between 1.5 and $4.5 \mathrm{~s}$, mean $=3 \mathrm{~s}$. There were six conditions in total: (i) Angry face - direct gaze; (ii) Angry face - averted gaze; (iii) Fearful face - direct gaze; (iv) Fearful face averted gaze; (v) Neutral face-direct gaze; (vi) Neutral face-averted gaze. Each image was presented four times, making a total of 40 trials in each condition. Total scanning time was $16.3 \mathrm{~min}$.

At the end of the scanning session participants completed a behavioral task outside the magnet. Here, they were presented with the face images used in the scanning study and asked to rate how angry each face appeared in one run, and how fearful each face appeared in a second run of the same images by pressing a key between 1 (not at all) and 9 (very). Images were presented for a maximum duration of $3000 \mathrm{~ms}$, or until the participant gave a response, with an ISI of $1000 \mathrm{~ms}$. The order of the rating runs (anger or fear) was counterbalanced across participants. In each run, each identity was presented once displaying each expression (anger, fear, neutral) with direct and averted gaze.

\section{IMAGING PARAMETERS}

MRI scanning was performed on a Siemens Tim Trio 3-Tesla MR scanner. Whole brain data were acquired with $\mathrm{T} 2{ }^{*}$-weighted echo-planar imaging (EPI) sensitive to BOLD signal contrast. Each image volume consisted of 40, 3-mm thick slices (gap 25\%; FOV $192 \times 192 \mathrm{~mm}$; voxel size $3 \times 3 \times 3 \mathrm{~mm}$; flip angle $78^{\circ}$; TE $30 \mathrm{~ms}$; TR $2424 \mathrm{~ms}$ ). Slices were acquired in an axial orientation. The first three volumes were discarded to allow for the effects of magnetic saturation. T1 weighted structural images were acquired at a resolution of $1 \times 1 \times 1 \mathrm{~mm}$.

\section{IMAGE ANALYSIS}

Data were analyzed using SPM 5 software (Wellcome Trust Centre for Neuroimaging, London, UK). Standard pre-processing was applied, including correction for slice-timing and head motion. Each participant's scans were normalized to the Montreal
Neurological Institute (MNI) - ICBM avg152 T1 weighted template, using $2 \mathrm{~mm}$ isotropic voxels and smoothed with a Gaussian kernel of 8-mm full-width half-maximum. Accuracy of registration and normalization procedures were individually checked for each participant, and all EPI images were inspected for signal dropout. Any participants with substantial signal dropout in the amygdala region were removed from the analysis. For each participant, individual events corresponding to each of the six conditions were modeled with a canonical hemodynamic response function. Realignment parameters were also included as effects of no interest to account for motion-related variance. A high pass filter of $128 \mathrm{~s}$ was used to remove low-frequency noise. A random effects analysis (one-sample $t$-test) was performed to analyze data at a group level. Single subject level contrasts were entered into a group-level regression analysis with anxiety as a covariate of interest. In this way, brain areas covarying with anxiety were identified using the same style of second-level SPM analysis applied to standard $t$-test or $F$-test comparisons. Regression plots showing the relationship between anxiety and contrast estimates for the maximal voxel are presented for information only. These data were not subject to secondary statistical analysis (Vul et al., 2009). The left and right amygdalae ROI's were defined using the Automatic Anatomical Labeling template (AAL) (Tzourio-Mazoyer et al., 2002), and small volume corrections were applied $(P<0.05)$. Other regions surviving $P<0.001$ uncorrected, minimum 10 contiguous voxels, are summarized in Tables 1-4 and Tables S1-S3 in Supplementary Material. All activations are reported using MNI coordinates.

\section{RESULTS}

\section{RATING EXPERIMENT}

First, we investigated the effect of gaze on the perceived intensity of emotional expression as measured in the rating task completed outside the magnet. A wilcoxon signed ranks test revealed that angry faces were rated as significantly more angry when their gaze was directed towards rather than away from the observer $(Z=2.17$, $P<0.05)$. Gaze had no effect on anger ratings for fearful or neutral faces $(P$ 's $>0.32)$. For fear ratings, there was a borderline trend showing that direct gaze fearful faces tended to be rated as more fearful than averted gaze fearful faces $(Z=1.70, P=0.08)$. In contrast, neutral faces were rated significantly more fearful when displaying averted gaze compared with direct gaze $(Z=2.85, P<0.005)$. Gaze had no effect on fear ratings of angry faces $(P>0.6)$. See Table 1.

Table 1 | Mean emotion intensity ratings ( $+S E$ ) for angry, fearful and neutral faces with direct and averted gaze used in the scanning experiment, and correlations (Spearman's rho) between state anxiety and intensity ratings relative to neutral comparison conditions (emotion minus neutral).

\begin{tabular}{lllll}
\hline Expression/Gaze & Anger rating (SE) & Correlation - state anxiety & Fear rating (SE) & Correlation - state anxiety \\
\hline Anger direct & $7.44(0.14)$ & $r=0.33^{*}$ & $3.25(0.41)$ & $r=-0.09$ \\
Anger averted & $7.29(0.15)$ & $r=0.22$ & $3.29(0.48)$ & $r=-0.07$ \\
Fear direct & $2.60(0.28)$ & $r=0.004$ & $7.11(0.22)$ & $r=0.32$ \\
Fear averted & $2.49(0.26)$ & $r=0.11$ & $6.98(0.22)$ & $r=0.39^{*}$ \\
Neutral direct & $2.03(0.18)$ & & $1.93(0.23)$ & \\
Neutral averted & $2.11(0.16)$ & & $2.17(0.23)$ & \\
\hline
\end{tabular}

${ }^{*} P<0.05$. 
To determine whether there was an interaction between gaze and emotion we computed an interaction term for the effect of gaze on ratings for anger and fear faces (direct anger - averted anger) $>$ (direct fear - averted fear) and performed a wilcoxon signed ranks test. This revealed no interaction between gaze and emotion $(Z=-0.8, P=0.42)$. To examine effects of gaze across emotion we also compared average ratings of direct anger plus direct fear against average ratings of averted anger plus averted fear (direct anger + direct fear $)>$ (averted anger + averted fear $)$. This revealed significantly increased ratings for direct gaze faces relative to averted gaze faces $(\mathrm{Z}=-2.47, P=0.01)$.

We also carried out a series of regressions (one-tailed) to investigate whether higher levels of anxiety were associated with increased ratings of anger and fear for averted and direct gaze faces (Table 1). In order to control for individual variation in use of the scale, we calculated intensity ratings for anger and fear faces relative to the appropriate neutral face condition rather than absolute ratings alone. This accords with the imaging analysis where the response to angry and fearful faces was determined relative to a neutral baseline. Anger ratings for direct gaze angry relative to direct gaze neutral faces showed a positive relationship with state anxiety $(r=0.33, P<0.05)$. However, we found no such pattern for averted angry faces relative to averted neutral faces $(P>0.1)$. Fear ratings for averted gaze fearful faces relative to averted gaze neutral faces were also found to increase as a function of state anxiety $(r=0.39, P<0.05)$, with a similar pattern observed for direct gaze fear relative to direct gaze neutral faces $(r=0.32, P=0.05)$. However, there was no relationship between state anxiety and ratings for within expression contrasts (e.g., direct anger vs. averted anger and direct fear vs. averted fear) $(P$ 's $>0.1)$. We also found no correlations between ratings of anger and fear faces and trait anxiety scores, although fear ratings for averted gaze neutral faces relative to direct gaze neutral faces did show a positive trend towards a relationship with trait anxiety $(P=0.08)$.

\section{BEHAVIORAL DATA}

For the gender discrimination task carried out during the scanning session, participants had a mean accuracy rate of $93.7 \% \pm 4.4 \%$ $S E$, and a mean response time of $715 \mathrm{~ms} \pm 19 \mathrm{~ms}$. A $3 \times 2$ (emotion $\times$ gaze) ANOVA for accuracy rates, revealed a main effect of emotion $[F(2,52)=16.93, P<0.001]$, with accuracy for angry faces $(90.4 \pm 1.2)$ slightly lower than those for fearful $(94.8 \pm 0.8)$ or neutral faces $(95.7 \pm 0.8)$. However, we found no effect of gaze $(F<1)$ on accuracy rates and no interaction between gaze and emotion $(F<1)$. Reduced accuracy for categorizing the gender of angry faces is consistent with previous research (Becker et al., 2007). For response times we also found a main effect of emotion $[F(2,52)=4.51$, $P<0.05]$. In this case, participants were faster to respond to neutral faces (705 ms \pm 20 SE) compared to angry $(720 \mathrm{~ms} \pm 20)$ or fearful faces ( $720 \mathrm{~ms} \pm 18)$. Again, there was no effect of gaze $(F<1)$ and no interaction between gaze and emotion $(F<1.5, P=0.25)$. It should be noted that the behavioral task (i.e., gender categorization) was orthogonal to the effect of interest (emotion $\times$ gaze).

\section{EFFECTS OF ANXIETY ON AMYGDALA RESPONSE}

Next, we investigated the influence of anxiety on the amygdala response to angry faces. First, we examined the relationship between anxiety and the amygdala response to angry facial expressions relative to their neutral comparison conditions. A regression analysis, including the contrast estimates for each participant, revealed a positive correlation with state anxiety in the right lateral amygdala for direct gaze angry faces compared to direct gaze neutral faces $(32,4,-26, z=3.12, P<0.02 \mathrm{svc}$ ) (Figure 2A). We noted that this cluster was located on the lateral border of the amygdala. To examine the reliability of these activations we also included a second independently defined amygdala ROI using anatomical masks based on the Talairach daemon database, defined using WFU Pick atlas software (Maldjian et al., 2003). Using this ROI we again found a positive correlation with state anxiety in the right lateral amygdala for direct gaze angry faces compared to direct gaze neutral faces $(30,0,-22, \mathrm{z}=2.71, P=0.05 \mathrm{svc})$. Thus, at least part of the activation fell within the amygdala as measured by two anatomical ROIs. By contrast, we found no correlation between anxiety and the amygdala response to averted gaze angry compared with averted gaze neutral faces $(P>0.11 \mathrm{svc})$.

Next, we examined the influence of anxiety on the amygdala response within expression (i.e., direct vs. averted angry faces) to verify that the increased response to direct anger, as a function of anxiety, could not be explained by a decreased response to direct neutral. Consistent with our predictions, we found a correlation with state anxiety in the extended amygdala when comparing direct gaze angry faces against averted gaze angry faces, with high-anxious individuals showing an increased response $(22,-6,-12, z=2.83$, $P<0.05$ svc) (Figure 2B). The locus of this activation is similar to that previously described as dorsal amygdala/substantia innominata (Whalen et al., 2001; Kim et al., 2003). See Table 2 for all activations correlated with state anxiety scores. A second analysis of the same data using the amygdala region as defined using the Pick atlas also showed that state anxiety predicted a significant interaction in the right dorsal amygdala $(22,-8,-10, z=2.86, P<0.03$ svc), and also showed a borderline effect in left amygdala $(-28,-6$, $-16, z=2.40, P=0.07)$.

Next, we investigated the amygdala response to facial signals of fear. State anxiety predicted an increased amygdala response to direct gaze fearful faces relative to direct gaze neutral faces in the right lateral amygdala border $(36,0,-24, z=3.13, P<0.02 \mathrm{svc})$. This activation appeared to lay outside of the amygdala, and subsequent analysis using the Pick Atlas ROI revealed no suprathreshold activations within the amygdala $(P=0.12 \mathrm{svc})$. Hence it is questionable whether this activation corresponds to the amygdala. We found that state anxiety was correlated with a bilateral amygdala response to averted gaze fearful faces relative to averted gaze neutral faces (Right amygdala: $18,-2,-16, z=3.13, P<0.02 \mathrm{svc}$; Left amygdala: -16 , $-6,-18, z=3.03, P<0.02 \mathrm{svc}$ ) (Figure 2C and Table 3). However, and in contrast to anger, we found no relationship between anxiety and the amygdala response when comparing direct and averted gaze fearful faces ( $P$ 's $>0.20 \mathrm{svc}$ ), demonstrating that the relationship between anxiety and the amygdala response to these two face categories did not significantly differ.

To test whether the differential effect of gaze and anxiety on the amygdala response to angry and fearful faces was statistically robust we explored the interaction between gaze and emotional expression [i.e. (direct gaze anger $>$ averted gaze anger) $>$ (direct gaze fear $>$ averted gaze fear)]. State anxiety predicted a significant interaction overlapping the right dorsal amygdala/SI $(24,-4,-12$, 
A

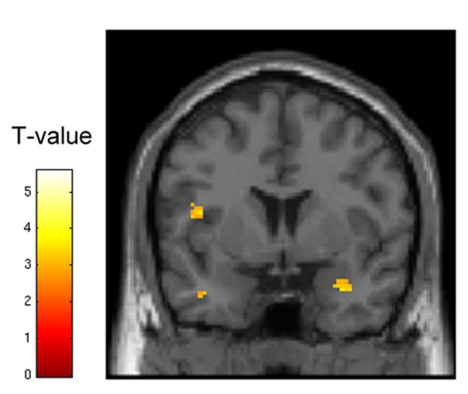

DIRECT ANGER > DIRECT NEUTRAL

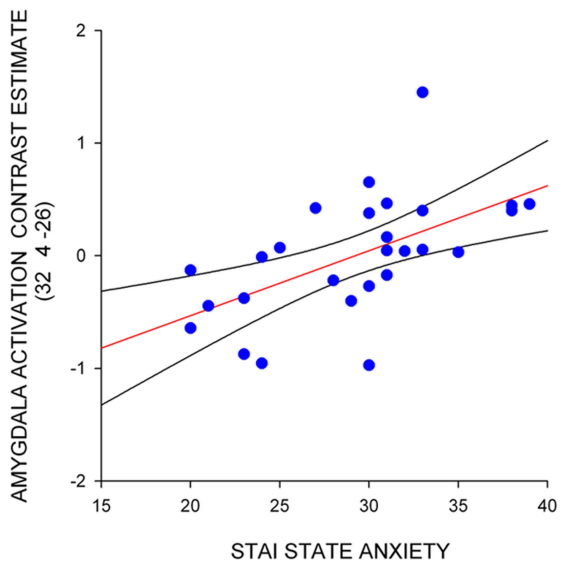

B

DIRECT ANGER > AVERTED ANGER
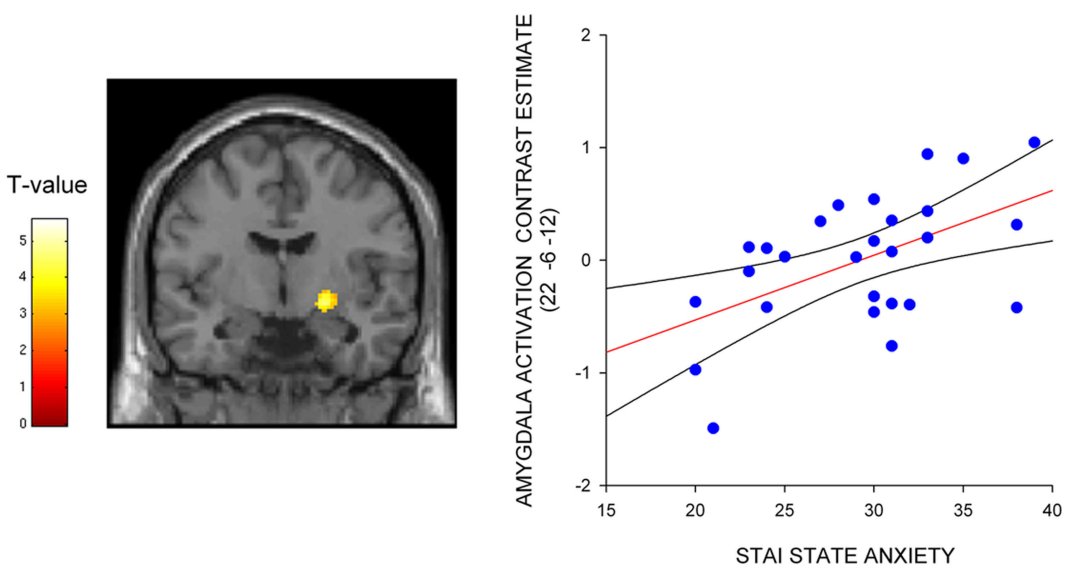

C AVERTED FEAR > AVERTED NEUTRAL
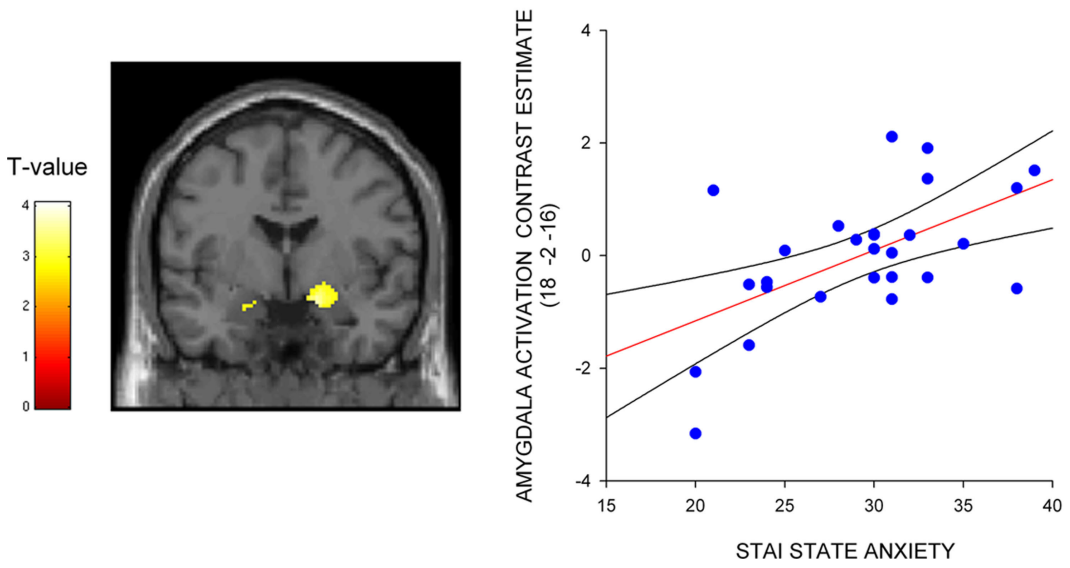

FIGURE 2 | Correlation between amygdala/SI activation and state anxiety shown on a coronal section of a canonical weighted T1 image for (A) direct gaze anger faces compared to direct gaze neutral faces (B) direct gaze angry faces compared to averted gaze angry faces, and (C) averted gaze fearful faces compared to averted gaze neutral faces. Scatter plots show contrast estimates of activation in the peak voxel of an anatomically defined amygdala $\mathrm{ROI}$ plotted against individual measures of state anxiety, included for illustration purposes only. All activation maps are thresholded at $P<0.005$ uncorrected (10 contiguous voxels) for display purposes. 
Table 2 | (A) Regions in which activation to angry faces showed a significant positive relationship with state anxiety scores. (B) Regions in which the interaction between emotional expression and gaze (anger, fear $\times$ direct, averted) showed a significant positive relationship with state anxiety scores.

\begin{tabular}{llllll}
\hline & \multicolumn{4}{c}{ MNI Coordinates } \\
\cline { 2 - 5 } Region & $x$ & $y$ & $z$ & Voxels & $Z$ \\
\hline
\end{tabular}

\begin{tabular}{|c|c|c|c|c|c|}
\hline \multicolumn{6}{|l|}{ A } \\
\hline \multicolumn{6}{|c|}{ Direct anger $>$ Averted anger } \\
\hline Right anterior insula & -42 & 8 & 12 & 820 & 4.34 \\
\hline $\begin{array}{l}\text { Left mid temporal } \\
\text { gyrus }\end{array}$ & -46 & -48 & -2 & 14 & 3.85 \\
\hline $\begin{array}{l}\text { Right amygdala/ } \\
\text { Substantia innominata }\end{array}$ & 22 & -6 & -12 & 10 & $2.83^{*}$ \\
\hline \multicolumn{6}{|c|}{ Direct anger $>$ Direct neutral } \\
\hline $\begin{array}{l}\text { Left superior temporal } \\
\text { sulcus }\end{array}$ & -50 & -48 & 12 & 537 & 4.45 \\
\hline $\begin{array}{l}\text { Right superior temporal } \\
\text { sulcus }\end{array}$ & 52 & -44 & 4 & 181 & 4.20 \\
\hline Superior frontal gyrus & -32 & 10 & 30 & 21 & 3.63 \\
\hline Right amygdala & 32 & 4 & -26 & 14 & $3.12 *$ \\
\hline \multicolumn{6}{|l|}{ B } \\
\hline \multicolumn{6}{|c|}{ (Direct anger $>$ Averted anger) $>$ (Direct fear $>$ Averted fear) } \\
\hline Left anterior/mid insula & -36 & 20 & 16 & 509 & 4.35 \\
\hline $\begin{array}{l}\text { Occipital cortex/primary } \\
\text { visual cortex }\end{array}$ & 4 & -84 & -22 & 173 & 3.89 \\
\hline $\begin{array}{l}\text { Left ventrolateral } \\
\text { prefrontal cortex }\end{array}$ & -52 & 38 & 4 & 152 & 3.65 \\
\hline $\begin{array}{l}\text { Right ventrolateral } \\
\text { prefrontal cortex }\end{array}$ & 44 & 54 & 6 & 13 & 3.41 \\
\hline Precentral gyrus & -8 & -22 & 72 & 49 & 3.37 \\
\hline $\begin{array}{l}\text { Right dorsolateral } \\
\text { prefrontal cortex }\end{array}$ & 24 & -52 & 10 & 21 & 3.35 \\
\hline $\begin{array}{l}\text { Right amygdala/ } \\
\text { Substantia innominata }\end{array}$ & 24 & -4 & -12 & 27 & $2.99 *$ \\
\hline
\end{tabular}

${ }^{*} P<0.05$ small volume corrected for amygdala ROI. All other activations are significant at $P<0.001$ whole brain uncorrected.

$z=2.99, P<0.03 \mathrm{svc}$ ) see Figure 3 and Table 2 . This cluster was also found to overlap the dorsal amygdala/SI using the Pick Atlas defined ROI $(22,-8,-10, z=2.77, P<0.03)$. Finally, consistent with the observation that averted gaze neutral faces were rated as more "fearful" than their direct gaze counterparts, we found a borderline significant relationship between state anxiety and activation in the right lateral amygdala border response to averted gaze neutral faces relative to direct gaze neutral faces $(32,4,-26, z=2.64, P=0.06 \mathrm{svc})$. However, we found no suprathreshold activation when using the Pick atlas defined ROI $(P>0.1 \mathrm{svc})$, suggesting that this activation lay just lateral to the amygdala. See Table 4 . The opposite contrast (direct neutral $>$ averted neutral) produced no significant amygdala response $(P>0.1 \mathrm{svc})$.

Since social anxiety and sex differences have also been shown to predict the amygdala response to angry faces (McClure et al., 2004; Phan et al., 2006) we examined the influence of these factors on amygdala activity. Neither were found to independently pre-
Table 3 | Regions in which activation to fearful faces showed a significant positive relationship with state anxiety scores.

\begin{tabular}{lccccc}
\hline & \multicolumn{5}{c}{ MNI coordinates } \\
\cline { 2 - 6 } Region & $\boldsymbol{x}$ & $\boldsymbol{y}$ & $\boldsymbol{z}$ & Voxels & $\boldsymbol{Z}$ \\
\hline DIRECT FEAR > DIRECT & NEUTRAL & & & \\
Superior frontal gyrus & 16 & 4 & 64 & 20 & 3.61 \\
Right mid temporal gyrus & 52 & -14 & -24 & 16 & 3.53 \\
Left mid temporal gyrus & -46 & 4 & -28 & 19 & 3.51 \\
Right amygdala & 36 & 0 & -24 & 18 & $3.13^{\text {a }}$ \\
AVERTED FEAR > AVERTED NEUTRAL & & & \\
Right amygdala & 18 & -2 & -16 & 36 & $3.13^{*}$ \\
Left amygdala & -16 & -6 & -18 & 5 & $3.03^{*}$ \\
AVERTED FEAR > DIRECT FEAR & & & & \\
Left inferior frontal gyrus & -40 & 32 & 4 & 115 & 4.10 \\
Precentral gyrus & 48 & 4 & 14 & 75 & 3.71 \\
Posterior parietal lobe & -10 & -46 & 68 & 35 & 3.51 \\
Primary visual cortex & 12 & -86 & -16 & 16 & 3.50 \\
\hline
\end{tabular}

${ }^{*} P<0.05$ small volume corrected for amygdala ROI. All other activations are significant at $P<0.001$ whole brain uncorrected. ${ }^{a}$ Activation did not survive small volume correction for Pick Atlas amygdala ROI.

dict the amygdala response, and all reported correlations remained significant after partialing out the effects of social anxiety (FNE) and sex differences (See Supplementary Material). In addition, consistent with the rating data, no significant correlations were found between trait anxiety scores and amygdala/SI activation, however a list of other regions showing a correlation with trait anxiety can be found in Table 3 in Supplementary Material. An additional analysis investigating hemispheric differences in the amygdala response to anger and fear is contained in Supplemental Materials. Results revealed that fear and anger did not differentially activate left and right amygdalae.

\section{OTHER BRAIN REGIONS}

It is also of interest that direct compared to averted gaze angry faces produced increased activation in the left mid/anterior insula that was strongly correlated with state anxiety $(-42,8,12, z=4.34, P<0.001$ uncorrected). This cluster overlapped with peak insula activation for the interaction between gaze and emotional expression, as modulated by anxiety [i.e. (direct anger $>$ averted anger) $>$ (direct fear $>$ averted fear)] $(-36,20,16, P<0.001$ uncorrected), with high-anxious individuals again showing a larger response to direct relative to averted gaze angry faces compared to direct relative to averted gaze fear faces. The insula region has been implicated in autonomic arousal (Critchley et al., 2002), thus activation in this region is consistent with the notion that angry faces directed at the observer are perceived as more threatening/arousing by high-anxious individuals. By contrast, we found no activation in the insula region for direct or averted fearful faces relative to their respective neutral face conditions.

\section{GROUP EFFECTS}

We were primarily interested in how the amygdala response to direct and averted gaze angry and fearful faces was modulated by anxiety. However, any correlation that may exist between 


\section{(DIRECT ANGER > AVERTED ANGER) > (DIRECT FEAR > AVERTED FEAR)}
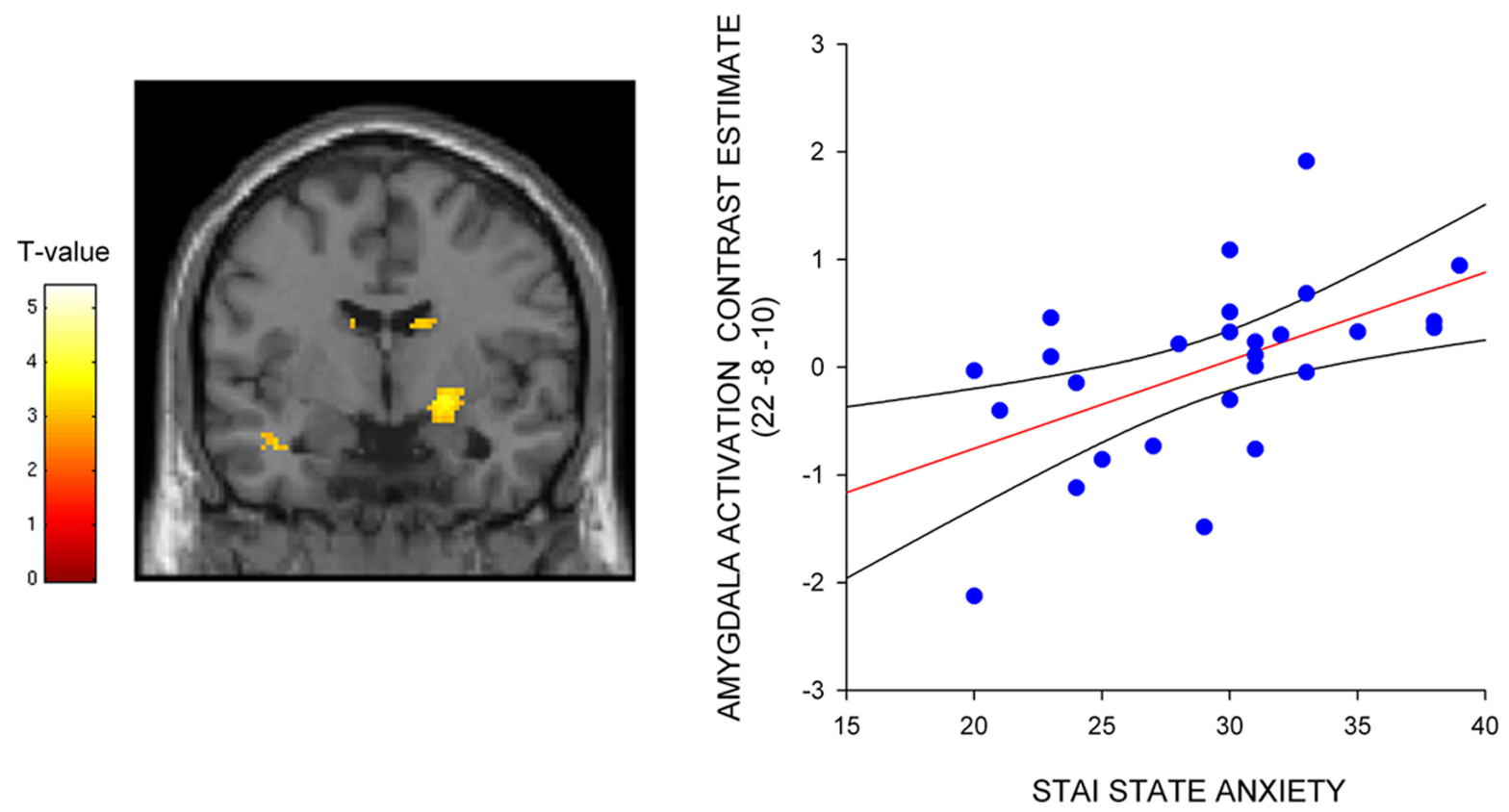

FIGURE 3 | Correlation between amygdala/SI activation and state anxiety for the interaction between gaze and expression - (direct gaze anger $>$ averted gaze anger) $>$ (direct gaze fear $>$ averted gaze fear), shown on a coronal section of a canonical weightedT1 image. Graph shows

contrast estimates of activation in the peak voxel of an anatomically defined amygdala ROI plotted against individual measures of state anxiety, included for illustration purposes only. Activation maps are thresholded at $P<0.005$ uncorrected (10 contiguous voxels) for display purposes.

the difference between two conditions for each participant and an additional variable (e.g., anxiety) is statistically distinct from the mean difference between two conditions. Hence, we also investigated the amygdala response to different emotional expressions across the group as a whole, independent of individual differences in anxiety. The only contrast producing an increase in activation was direct gaze fearful faces compared with averted gaze fearful faces, which produced a significantly increased response in the left amygdala $(-22,-8,-16, z=3.07$, $P<0.05$ svc). In addition, when collapsing across gaze direction we found no main effect of anger or fear relative to neutral faces $(P$ 's $>0.25)$. Thus, consistent with previous research investigating the amygdala response to facial signals of threat in subjects spanning a wide range of anxiety scores (Bishop et al., 2004; Ewbank et al., 2009c), mean effects were not apparent when comparing responses across a range of anxiety scores, due to differences in anxiety producing relative increases and decreases in the amygdala response to facial signals of anger and fear. The one exception was the comparison of direct gaze fear versus averted gaze fear faces.

\section{DISCUSSION}

Our results demonstrate that the response in the extended amygdala to facial signals of anger and fear is differentially modulated by changes in gaze direction and individual levels of anxiety. Specifically, the interaction between expression (anger/ fear) and gaze (direct/averted), in the right dorsal amygdala/SI, showed a correlation with state anxiety. High-anxious individuals showed a greater response to direct gaze angry faces compared to averted gaze angry faces, while the response to fearful faces increased as a function of anxiety for both direct and averted gaze. Moreover, these effects persisted despite controlling for the influence of social anxiety and sex, indicating that our effects cannot be attributed to these other factors that are known to affect amygdala function.

The observed dorsal amygdala/SI activation is spatially similar to that reported in previous studies addressing the role of the amygdala in processing threat-related stimuli (Phelps et al., 2001; Whalen et al., 2001; Kim et al., 2003; Pessoa et al., 2006). The SI is characterized as a functional and anatomical continuum of the central and medial nucleus of the amygdala (Heimer et al., 1997; Heimer, 2003), and has been proposed to play a role in coding the salience or arousal value of a face (Kim et al., 2003; Davis et al., 2009). Not all correlations were found in the dorsal extended amygdala. However, due to the spatial resolution of the current study, we hesitate to make strong assertions regarding the spatial localization of associated with different contrasts, and note that both dorsal and lateral sections of the amygdala have been reported for facial expressions of fear and anger in previous studies (Whalen et al., 2001; Etkin et al., 2004; Sato et al., 2004; Ewbank et al., 2009c). On an additional note, unlike previous work directly comparing angry and fearful faces (Whalen et al., 2001) we did not find a differential response between these two emotions at a group level within the amygdala. However, Whalen et al's. (2001) results were 
Table 4 | Regions in which activation to neutral faces showed a significant positive relationship with state anxiety scores. All activations are significant at $P<0.001$ whole brain uncorrected.

\begin{tabular}{|c|c|c|c|c|c|}
\hline \multirow[b]{2}{*}{ Region } & \multicolumn{5}{|c|}{ MNI Coordinates } \\
\hline & $x$ & $y$ & $z$ & Voxels & $\mathbf{Z}$ \\
\hline \multicolumn{6}{|c|}{ AVERTED NEUTRAL > DIRECT NEUTRAL } \\
\hline $\begin{array}{l}\text { Right middle-temporal } \\
\text { gyrus }\end{array}$ & 50 & -22 & -20 & 62 & 4.32 \\
\hline Cuneus & 10 & -78 & 30 & 577 & 4.23 \\
\hline $\begin{array}{l}\text { Left inferior parietal } \\
\text { lobule/superior } \\
\text { temporal sulcus }\end{array}$ & -52 & -42 & 24 & 1002 & 4.20 \\
\hline $\begin{array}{l}\text { Right inferior frontal } \\
\text { gyrus }\end{array}$ & 52 & 26 & -6 & 25 & 4.04 \\
\hline Precuneus & -12 & -56 & 44 & 123 & 3.74 \\
\hline Middle frontal gyrus & 50 & 8 & 44 & 93 & 3.64 \\
\hline $\begin{array}{l}\text { Dorsomedial prefrontal } \\
\text { cortex }\end{array}$ & 24 & -52 & 10 & 44 & 3.60 \\
\hline $\begin{array}{l}\text { Right inferior parietal } \\
\text { lobule }\end{array}$ & 70 & -36 & 22 & 317 & 3.58 \\
\hline $\begin{array}{l}\text { Left anterior superior } \\
\text { temporal sulcus }\end{array}$ & -54 & -10 & -12 & 17 & 3.53 \\
\hline Medial frontal gyrus & 14 & 2 & 66 & 14 & 3.40 \\
\hline Postcentral gyrus & -20 & -40 & 60 & 25 & 3.39 \\
\hline $\begin{array}{l}\text { Left temporoparietal } \\
\text { junction }\end{array}$ & -46 & -70 & 18 & 16 & 3.31 \\
\hline $\begin{array}{l}\text { Occipital cortex/primary } \\
\text { visual cortex }\end{array}$ & -10 & -96 & 2 & 18 & 3.26 \\
\hline Right amygdala & 32 & 4 & -26 & 20 & $2.64^{*}$ \\
\hline
\end{tabular}

${ }^{*} P=0.06$ svc; activation did not survive small volume correction for Pick Atlas amygdala $\mathrm{ROI}$.

found with high resolution imaging, and future research should explore whether the relationship between anxiety and the neural response to fear and anger can also be differentiated at a higher spatial resolution.

It is also of note that the effects of anxiety on the neuroimaging data were mirrored by a similar pattern in the behavioral ratings data, for which ratings of direct, but not averted gaze angry faces were also found to correlate with state anxiety. By contrast, fearful ratings of both averted and direct gaze fearful faces were correlated with state anxiety, paralleling the finding that the relationship between the amygdala response to fearful expressions and anxiety was less dependent on gaze direction. Further analysis indicated that the amygdala response to angry and fearful faces showed a more reliable relationship with anxiety than with subjective ratings of emotional intensity (see Supplementary Material). In other words, while anxiety was correlated was both subjective ratings and the amygdala response, the amygdala response was best accounted for by variation in anxiety rather than an index of subjects' subjective evaluation of the stimuli.

Previously, we demonstrated that the observers' focus of attention differentially modulates the amygdala response to angry and fearful faces (Ewbank et al., 2009c). High-anxious individuals showed an increased amygdala response to fearful faces presented inside or outside the focus of the observers' attention, and no significant difference between the two conditions. By contrast, the amygdala response to angry faces was restricted to the attended condition (Ewbank et al., 2009c), as evidenced by an increased response to attended versus unattended angry expressions as a function of anxiety. Here, we show that the expresser's direction of attention predicts a similar differential neural response that is also correlated with anxiety. In the previous study the relationship between anxiety and the amygdala response to fearful faces was more marked in the unattended condition. In the current study we also found a more reliable relationship between anxiety and activity in the amygdala to averted gaze fearful faces than to direct gaze fearful faces. However, across both studies, the amygdala response to fearful faces was not significantly affected by the paticipant's focus of attention or the face's gaze direction. In contrast, both attention and gaze direction modulated the amygdala response to angry faces.

Our findings support the role of the amygdala and extended amygdala in coding the faces' perceived level of threat, and the more general proposal that the amygdala codes the relevance or significance of a stimulus to the observer (Sander et al., 2003, 2005; Ewbank et al., 2009a; Adolphs, 2010). Thus, direct gaze angry faces would be considered of greater relevance to high-anxious individuals than low-anxious individuals. Indeed, in their account of the amygdala's role as a "relevance detector", Sander and colleagues predict that the amygdala should show greater activation to angry faces when the gaze is directed towards, rather than away from the observer (Sander et al., 2003). Aversive conditioning research using angry faces as the conditioned stimulus has also reported more resilience to extinction when the faces' gaze is directed towards rather than away from the observer (Dimberg and Ohman, 1983). Similarly, as we have discussed, numerous behavioral studies have also demonstrated that direct gaze enhances the threat value of angry faces, relative to averted gaze. In contrast, behavioral data has found less consistent effects of gaze on perceived threat or intensity of fearful faces, with some showing that averted gaze enhances perception of fear (Adams and Kleck, 2003, 2005; Sander et al., 2007), and others finding the opposite or no effect of gaze, including our own (Adams and Kleck, 2005; Graham and LaBar, 2007; Hess et al., 2007; Bindemann et al., 2008). Furthermore, a differential effect of gaze and anxiety on the amygdala response to angry, but not fearful faces, is also consistent with recent work indicating that effects of direct gaze on the processing of angry faces is disrupted in patients with amygdala lesions, while the effect of gaze on processing fearful faces is relatively unchanged (Cristinzio et al., 2010).

Our current finding for angry faces also accords with previous evidence indicating an increased amygdala response to peripherally presented angry expressions displayed by heads that were oriented towards rather than away from the observer (Sato et al., 2004). However, our current study shows that, in high-anxious individuals, the effect of gaze direction on amygdala/SI response is specific to the viewing of angry faces, rather than other, high arousal expressions, such as fear, for which the amygdala response is significantly less affected by gaze direction. Furthermore, we demonstrate that the relationship between anxiety and amygdala activation to both angry and fearful faces mirrors the relationship between subjective ratings of emotional intensity portrayed by the faces and individual measures of state anxiety. 
The amygdala response to facial signals of anger and fear as a function of anxiety appears inconsistent with the previous finding of increased amygdala activation to direct gaze fearful faces and averted gaze angry faces (ambiguous threat) relative to averted gaze fearful and direct gaze angry faces (unambiguous threat) (Adams et al., 2003). However, consistent with Adams et al. (2003) our group-based comparisons, irrespective of individual differences in anxiety, showed that direct gaze fearful faces produced greater amygdala activation than averted gaze fearful faces. By contrast, we found no evidence of a corresponding increase in amygdala activation to averted relative to direct gaze angry faces. Other fMRI studies have also found partly similar or different results to those of Adams et al. (2003) and the group-based effects in the current study. Hadjikhani et al. (2008) found an increased amygdala response to averted gaze fearful faces relative to direct gaze fearful faces. Also, Straube et al. (2010) reported greater amygdala response to averted gaze faces displaying anger, joy and neutral expressions versus direct gaze exemplars of the same expressions, but no gaze $\times$ expression interaction, indicating a generalized effect of averted versus direct gaze regardless of valence. However, they found no difference in ratings of threat or arousal for direct and averted angry faces; a result that appears inconsistent with previous studies on the interaction between gaze and angry expressions.

One possible explanation for the difference between the different studies was identified Bindemann et al. (2008), who found that the influence of gaze on the perception of fearful faces varied according to the stimulus set used and task demands. On a similar note, previous work has demonstrated that the relative salience of gaze and expression may be an important factor in influencing the extent to which the two variables are integrated (Ganel et al., 2005). However, while it is possible that differences in speed of processing could help explain the discrepancy between different behavioral and fMRI studies, an additional series of experiments that go beyond the scope of the current study would be required to verify that this is the case. Another potential explanation for differences is that Adams and colleagues extracted the neural response to angry and fearful faces using a functional definition of the amygdala (i.e., fearful faces versus baseline). Thus, they restricted their analysis to a specific region of the amygdala, rather than the amygdala per se. We therefore performed an additional analysis using a similar functional localizer, but in contrast to Adams et al. (2003) we still found no increase to ambiguous relative to unambiguous threat [i.e. (anger averted and fear direct) $>$ (anger direct and fear averted), $F<1$ ] (Supplementary Materials). A third alternative explanation is that individual differences in relevant personality dimensions, such as anxiety, contribute to the inconsistent findings among studies. Considered as separate groups (i.e., median split), our findings suggest that the low-anxious individuals show a trend towards effects that accord more closely with those of Adams et al. (2003) (i.e., increased response to direct fear $>$ averted fear and averted anger $>$ direct anger), whereas high-anxious participants show the opposite pattern for anger and no influence of gaze for fear (see Supplemental Materials). However, our study was designed to examine anxiety as a continuous variable and was not optimized for examining differences between high and low-anxious groups, where the upper and lower extremes of the non-clinical anxiety range (e.g., upper and lower 25th percentiles) should be compared. Thus, although it would be premature to conclude that inconsistencies in the literature can be explained by qualitatively distinct patterns of amygdala activation in high and low-anxious groups, this should be explored in further groupbased studies. Despite this, these patterns suggest that inconsistent findings may be partly due to differences in the anxiety levels of the sample used. However, variation in other personality dimensions is also likely to be important.

It is also relevant that correlations with anxiety were not restricted to the amygdala and extended amygdala. In particular, the neural response in the $\mathrm{mid} /$ anterior insula to direct relative to averted gaze angry faces was correlated with anxiety. Although this region has often been implicated in the recognition of facial expressions of disgust (Phillips et al., 1997; Calder et al., 2000, 2001; von dem Hagen et al., 2009), Calder and colleagues have suggested that this is unlikely to constitute a "disgust specific" region, but rather is involved in coding a more general dimension on which disgust loads particularly highly (Calder, 2003; Calder et al., 2007). Indeed, the insula is thought to play an important role in autonomic arousal, including the anticipation of pain (Ploghaus et al., 1999), and activity in this region is correlated with physiological changes in skin conductance and heart rate (Critchley et al., 2000a,b). Further work also demonstrates that the insula shows an increased response to both facial and vocal signals of anger (Strauss et al., 2005; Quadflieg et al., 2008). Thus, increased insula activation to aggressive faces directed towards rather than away from the observer, is consistent with the observation that they are perceived as more threatening; an effect that is amplified in high-anxious individuals.

It is also worth considering that correlations between the amygdala response and anxiety measures were found for state but not trait anxiety. Consistent with this, a recent meta-analysis suggests greater effect sizes when using state anxiety in within group studies (Bar-Haim et al., 2007). A state anxiety driven response may also be consistent with the role of the amygdala as a central component of a "fear- or threat-relevance" system. However, earlier work found that both state and trait anxiety predicted the amygdala response to angry and fearful faces (Bishop et al., 2004; Etkin et al., 2004; Ewbank et al., 2009c). Thus, while it is possible that state may be a more reliable predictor of the amygdala response, this requires additional investigation, not least because the two measures are correlated.

In the current study, group-level comparisons did not show any difference in amygdala activation to direct or averted gaze angry or fearful faces relative to neutral faces. This result is perhaps unsurprising considering that one meta-analysis found that $40 \%$ of studies fail to find a significant amygdala response to fearful versus neutral expressions (Murphy et al., 2003). Instead, our findings suggest that a substantial degree of variability in the amygdala response to threat may have a meaningful psychological basis, and that consideration of relevant factors (e.g., anxiety) can reveal consistent patterns of activation that are not apparent when using group-level contrasts. It is noteworthy that similar effects have been found in a number of other studies addressing the role of other personality dimensions (Canli et al., 2002; Beaver et al., 2008; Passamonti et al., 2008). 
In conclusion, our results indicate that the amygdala/extended amygdala response to angry and fearful faces is influenced by the direction of gaze of the expresser, and the participants' level of selfreported anxiety. High-anxious individuals showed an increased amygdala/SI response to angry faces only when the gaze was directed towards them. By contrast, anxiety predicted an increased amygdala response to fearful faces that was less dependent on gaze direction. Our findings accord with the role of the amygdala as a "relevance detector" (Sander et al., 2003) and appear difficult to reconcile with the amygdala's proposed role in coding ambiguous stimuli (Davis and Whalen, 2001), which has more difficulty in accounting for effects of anxiety. Moreover, the different patterns of response observed to fear and anger as a function of anxiety accord with the different nature of threat signaled by these two expressions. The perceived threat, and hence relevance of an angry face is greatest when

\section{REFERENCES}

Adams, R. B., Gordon, H. L., Baird, A. A., Ambady, N., and Kleck, R. E. (2003). Effects of gaze on amygdala sensitivity to anger and fear faces. Science 300 , 1536.

Adams, R. B., and Kleck, R. E. (2003). Perceived gaze direction and the processing of facial displays of emotion. Psychol. Sci. 14, 644-647.

Adams, R. B., and Kleck, R. E. (2005). The effects of direct and averted gaze on the perception of facially communicated emotion. Emotion 5, 3-11.

Adolphs, R. (1999). The human amygdala and emotion. Neuroscientist 5, 125-137.

Adolphs, R. (2010). What does the amygdala contribute to social cognition? Ann. N. Y. Acad. Sci. 1191, 42-61.

Averill, J. R. (1982). Anger and Aggression: An Essay on Emotion. New York: Springer-Verlag.

Bar-Haim, Y., Lamy, D., Pergamin, L., Bakermans-Kranenburg, M. J., and van Ijzendoorn, M. H. (2007). Threatrelated attentional bias in anxious and nonanxiousindividuals: a meta-analytic study. Psychol. Bull. 133, 1-24.

Beaver, J. D., Lawrence, A. D., Passamonti, L., and Calder, A. J. (2008). Appetitive motivation predicts the neural response to facial signals of aggression. J. Neurosci. 28, 2719-2725.

Becker, D. V., Kenrick, D. T., Neuberg, S. L., Blackwell, K. C., and Smith, D. M. (2007). The confounded nature of angry men and happy women. $J$. Pers. Soc. Psychol. 92, 179-190.

Bindemann, M., Burton, A. M., and Langton, S. R. H. (2008). How do eyegaze and facial expression interact? Vis. cogn. 16, 708-733.

Bishop, S. J., Duncan, J., and Lawrence, A. D. (2004). State anxiety modulation of the amygdala response to unattended threat-related stimuli. J. Neurosci. 24, 10364-10368.
Blanchard, R. J., and Blanchard, D. C. (2003). What can animal aggression research tell us about human aggression? Horm. Behav. 44, 171-177.

Calder, A. J. (2003). Disgust discussed. Ann. Neurol. 53, 427-428.

Calder, A. J., Beaver, J. D., Davis, M. H., van Ditzhuijzen, J., Keane, J., and Lawrence, A. D. (2007). Disgust sensitivity predicts the insula and pallidal response to pictures of disgusting foods. Eur. J. Neurosci. 25, 3422-3428.

Calder, A. J., Keane, J., Manes, F., Antoun, N., and Young, A. W. (2000). Impaired recognition and experience of disgust following brain injury. Nat. Neurosci. 3, 1077-1078.

Calder, A. J., Lawrence, A. D., and Young, A.W. (2001). The neuropsychology of fear and loathing. Nat. Rev. Neurosci. 2, 352-363.

Calder, A. J., Young, A. W., Rowland, D., Perrett, D. I., Hodges, J. R., and Etcoff, N. L. (1996). Facial emotion recognition after bilateral amygdala damage: differentially severe impairment of fear. Cogn. Neuropsychol. 13, 699-745.

Canli, T., Sivers, H., Whitfiled, S. L., Gotlib, I. H., and Gabrieli, J. D. E. (2002). Amygdala response to happy faces as a function of extraversion. Science 295, 2191.

Carleton, R. N., Collimore, K. C., and Asmundson, G. J. (2007). Social anxiety and fear of negative evaluation: construct validity of the BFNE-II. $J$ Anxiety Disord 21, 131-141.

Carleton, R. N., McCreary, D. R., Norton, R. J., and Asmundson, G. J. G. (2006). Brief fear of negative evaluation scale - revised. Depress. Anxiety 23, 297-303.

Cristinzio, C., N’Diaye, K., Seeck, M., Vuilleumier, P., and Sander, D. (2010). Integration of gaze direction and facial expression in patients with unilateral

attended by and directed towards the observer. By contrast, the threat and relevance signaled by a fearful face is significantly less dependent on the expresser's or the observer's direction of attention.

\section{ACKNOWLEDGMENTS}

This research was funded by the UK Medical Research Council under project code U.1055.02.001.0001.01 (Andrew J. Calder). We wish to thank the CBU radiographers for their assistance in this project, Chris Ashwin for his help in adapting the stimuli and Lauri Nummenmaa for his help in data acquisition.

\section{SUPPLEMENTARY MATERIAL}

The Supplementary Material for this article can be found online at http://www.frontiersin.org/neuroscience/humanneuroscience/ paper/10.3389/fnhum.2010.00056/

amygdala damage. Brain 133(Pt 1), 248-261.

Critchley, H. D., Corfield, D. R., Chandler, M. P., Mathias, C. J., and Dolan, R. J. (2000a). Cerebral correlates of autonomic cardiovascular arousal: a functional neuroimaging investigation in humans. J. Physiol. 523(Pt 1), 259-270.

Critchley, H. D., Elliott, R., Mathias, C. J., and Dolan, R. J. (2000b). Neural activity relating to generation and representation of galvanic skin conductance responses: a functional magnetic resonance imaging study. J. Neurosci. 20, 3033-3040.

Critchley, H. D., Mathias, C. J., and Dolan, R. J. (2002). Fear conditioning in humans: the influence of awareness and autonomic arousal on functional neuroanatomy. Neuron 33, 653-663.

Davis, F. C., Johnstone, T., Mazzulla, E. C., Oler, J. A., and Whalen, P. J. (2009). Regional response differences across the human amygdaloid complex during social conditioning. Cereb. Cortex. 20, 612-621.

Davis, M., and Whalen, P. J. (2001). The amygdala: vigilance and emotion. $\mathrm{Mol}$. Psychiatry 6, 13-34.

Dimberg, U., and Ohman, A. (1983). The effects of directional facial cues on electrodermal conditioning to facial stimuli. Psychophysiology 20, 160-167.

Ekman, P., and Friesen, W. V. (1975). Unmasking the Face: A Guide to Recognizing Emotions from Facial Clues Englewood Cliffs, NJ: Prentice Hall.

Etkin, A., Klemenhagen, K. C., Dudman, J. T., Rogan, M. T., Hen, R., Kandel, E. R., and Hirsch, J. (2004). Individual differences in trait anxiety predict the response of the basolateral amygdala to unconsciously processed fearful faces. Neuron 44, 1043-1055.

Evans, K. C., Wright, C. I., Wedig, M. M., Gold, A. L., Pollack, M. H., and
Rauch, S. L. (2007). A functional MRI study of amygdala responses to angry schematic faces in social anxiety disorder. Depress. Anxiety 25, 496-505.

Ewbank, M. P., Barnard, P. J., Ramponi, C., Croucher, C. J., and Calder, A. J. (2009a). The amygdala response to images with impact. Soc. Cogn. Affect. Neurosci. 4, 127-133.

Ewbank, M. P., Fox, E., and Calder, A. J. (2009b). Why are you angry with me? Facial expressions of threat influence gaze perception. $J$ Vis $9,1-7$.

Ewbank, M. P., Lawrence, A. D., Passamonti, L., Keane, J., Peers, P. V., and Calder, A. J. (2009c). Anxiety predicts a differential neural response to attended and unattended facial signals of anger and fear. Neuroimage 44, 1144-1151.

Fox, E., Mathews, A., Calder, A. J., and Yiend, J. (2007). Anxiety and sensitivity to gaze direction in emotionally expressive faces. Emotion 7, 478-486.

Ganel, T., Goshen-Gottstein, Y., and Goodale, M. A. (2005). Interactions between the processing of gaze direction and facial expression. Vision Res. 45, 1191-1200.

Graham, R., and LaBar, K. (2007). Garner interference reveals dependencies between emotional expression and gaze in face perception. Emotion 7 , 296-313.

Hadjikhani, N., Hoge, R., Snyder, J., and de Gelder, B. (2008). Pointing with the eyes: the role of gaze in communicating danger. Brain Cogn. 68, 1-8.

Heimer, L. (2003). A new anatomical framework for neuropsychiatric disorders and drug abuse. Am. J. Psychiatry 160, 1726-1739.

Heimer, L., Harlan, R. E., Alheid, G. F., Garcia, M. M., and de Olmos, J. (1997). Substantia innominata: a notion which impedes clinical-anatomical correlations in neuropsychiatric disorders. Neuroscience 76, 957-1006. 
Hess, U., Adams, R. B., and Kleck, R. E. (2007). Looking at you or looking elsewhere: The influence of head orientation on the signal value of emotional facial expressions. Motiv. Emot. 31, 137-144.

Kim, H., Somerville, L. H., McClean, A. A., Johnstone, T., Shin, L. M., and Whalen, P. J. (2003). Functional MRI responses of the human dorsal amygdala/substantia innominata region to facial expressions of emotion. Ann. N. Y. Acad. Sci. 985, 533-535.

Lundqvist, D., and Litton, J.-E. (1998). The Averaged Karolinska Directed Emotional Faces-AKDEF. Stockholm: AKDEFCD ROM. Psychology section, Karolinska Institutet.

Maldjian, J. A., Laurienti, P. J., Kraft, R. A., and Burdette, J. H. (2003). An automated method for neuroanatomic and cytoarchitectonic atlas-based interrogation of $\mathrm{AMRI}$ data sets. Neuroimage 19, 1233-1239 (WFU Pickatlas, version 1232.1234).

McClure, E. B., Monk, C. S., Nelson, E. E., Zarahn, E., Leibenluft, E., Bilder, R. M., Charney, D. S., Ernst, M., and Pine, D. S. (2004). A developmental examination of gender differences in brain engagement during evaluation of threat. Biol. Psychiatry 55, 1047-1055.

Murphy, F. C., Nimmo-Smith, I., and Lawrence, A. D. (2003). Functional neuroanatomy of emotion: A metaanalysis. Cogn. Affect. Behav. Neurosci. 3, 207-233.

Passamonti, L., Rowe, J. B., Ewbank, M., Hampshire, A., Keane, J., and Calder, A. J. (2008). Connectivity from the ventral anterior cingulate to the amygdala is modulated by appetitive motivation in response to facial signals of aggression. Neuroimage 43, 562-570.

Pessoa, L., Japee, S., Sturman, D., and Ungerleider, L. G. (2006). Target visibility and visual awareness modulate amygdala responses to fearful faces. Cereb. Cortex 16, 366-375.
Phan, K. L., Fitzgerald, D. A., Nathan, P. J., and Tancer, M. E. (2006). Association between amygdala hyperactivity to harsh faces and severity of social anxiety symptoms in generalized social phobia. Biol. Psychiatry 59, 421-429.

Phelps, E. A., Ling, S., and Carrasco, M. (2006). Emotion facilitates perception and potentiates the perceptual benefits of attention. Psychol. Sci. 17, 292-299.

Phelps, E. A., O’Connor, K. J., Gatenby, J. C., Gore, J. C., Grillon, C., and Davis, M. (2001). Activation of the left amygdala to a cognitive representation of fear. Nat. Neurosci. 4, 437-441.

Phillips, M. L., Young, A. W., Senior, C., Brammer, M., Andrew, C., Calder, A. J., Bullmore, E. T., Perrett, D. I., Rowland, D., Williams, S. C. R., Gray, J. A., and David, A. S. (1997). A specific neural substrate for perceiving facial expressions of disgust. Nature 389, 495-498.

Ploghaus, A., Tracey, I., Gati, J. S., Clare, S., Menon, R. S., Matthews, P. M., and Rawlings, J. N. (1999). Dissociating pain from its anticipation in the human brain. Science 284, 1979-1981.

Putman, P., Hermans, E., and van Honk, J. (2006). Anxiety meets fear in perception of dynamic expressive gaze. Emotion 6, 94-102.

Quadflieg, S., Mohr, A., Mentzel, H. J., Miltner,W.H., and Straube, T. (2008). Modulation of the neural network involved in the processing of anger prosody: the role of task-relevance and social phobia. Biol. Psychol. 78, 129-137.

Sander, D., Grafman, J., and Zalla, T. (2003). The human amygdala: an evolved system for relevance detection. Rev. Neurosci. 14, 303-316.

Sander,D., Grandjean, D., Kaiser, S., Wehrle, T., and Scherer, K. R. (2007). Interaction effects of perceived gaze direction and dynamic facial expression: evidence for appraisal theories of emotion. Eur. J. Cogn. Psychol., 19, 470-480.
Sander, D., Grandjean, D., Pourtois, G. Schwartz, S., Seghier, M. L., Scherer, K. R., and Vuilleumier, P. (2005). Emotion and attention interactions in social cognition: Brain regions involved in processing anger prosody. Neuroimage 28, 848-858.

Sato, W., Yoshikawa, S., Kochiyama, T. and Matsumura, M. (2004). The amygdala processes the emotional significance of facial expressions: an fMRI investigation using the interaction between expression and face direction. Neuroimage 22, 1006-1013.

Spielberger, C. D. (1983). Manual for the State-Trait Anxiety Inventory (STAI). PaloAlto, CA: Consulting Psychologists Press.

Straube, T., Langohr, B., Schmidt, S., Mentzel, H. J., and Miltner, W. H. (2010). Increased amygdala activation to averted versus direct gaze in humans is independent of valence of facial expression. Neuroimage 49, 2680-2686.

Strauss, M. M., Makris, N., Aharon, I., Vangel, M. G., Goodman, J., Kennedy, D. N., Gasic, G. P., and Breiter, H. C. (2005). fMRI of sensitization to angry faces. Neuroimage 26, 389-413.

Susskind, J.M., Lee, D.H., Cusi,A., Feiman, R., Grabski, W., and Anderson, A. K. (2008). Expressing fear enhances sensory acquisition. Nat. Neurosci. $11,843-850$.

Tipples, J. (2006). Fear and fearfulness potentiate automatic orienting to eye gaze. Cogn. Emot. 20, 309-320.

Tottenham, N., Tanaka, J., Leon, A. C. McCarry, T., Nurse, M., Hare, T. A. Marcus, D. J., Westerlund, A., Casey, B. J., and Nelson, C. (2009). The NimStim set of facial expressions: judgments from untrained research participants. Psychiatry Res 168, 242-249.

Tzourio-Mazoyer, N., Landeau, B. Papathanassiou, D., Crivello, F., Etard, O., Delcroix, N., Mazoyer, B., and Joliot, M. (2002). Automatic anatomical labelling of activations in SPM using a macroscopic anatomical parcellation of the MNI MRI single-subject brain Neuroimage 15, 273-289.

Van Casteren, M., and Davis, M. H. (2006). Mix, a program for pseudorandomization. Behav. Res. Methods 38, 584-589.

von dem Hagen, E., Beaver, J. D., Ewbank, M. P., Keane, J., Passamonti, L., Lawrence, A. D., and Calder, A. J. (2009). Leaving a bad taste in your mouth but not in my insula. Soc. Cogn. Affect. Neurosci. 4, 379-386.

Vul, E., Harris, C., Winkielman, P., and Pashler, H. (2009). Puzzlingly high correlations in fMRI studies of emotion, personality, and social cognition. Perspect. Psychol. Sci. 4, 274-290.

Whalen, P. J. (2007). The uncertainty of it all. Trends Cogn. Sci. 11, 499-500.

Whalen, P. J., Shin, L. M., McInerney, S. C., Fischer, H., Wright, C. I., and Rauch, S. L. (2001). A functional MRI study of human amygdala responses to facial expressions of fear vs. anger. Emotion $1,70-83$.

Conflict of Interest Statement: The authors declare that the research was conducted in the absence of any commercial or financial relationships that could be construed as a potential conflict of interest.

Received: 17 December 2009; paper pending published: 16 February 2010; accepted: 11 June 2010; published online: 07 July 2010. Citation: Ewbank MP, Fox E and Calder AJ (2010) The interaction between gaze and facial expression in the amygdala and extended amygdala is modulated by anxiety. Front. Hum. Neurosci. 4:56. doi: 10.3389/fnhum.2010.00056

Copyright (c) 2010 Ewbank, Fox and Calder. This is an open-access article subject to an exclusive license agreement between the authors and the Frontiers Research Foundation, which permits unrestricted use, distribution, and reproduction in any medium, provided the original authors and source are credited. 